\title{
On Partial Order Characterizations of Information Structures*
}

\begin{abstract}
Demosthenis Teneketzis $^{\dagger}$ and Mark S. Andersland ${ }^{\ddagger}$
Abstract. The degree of concurrency possible in an informationally decentralized nonsequential system is determined by the precedence constraints imposed by the system's information structure on its controlled and uncontrolled inputs. One approach to characterizing these constraints is to attempt to associate each realization of the system's inputs with the strongest partial ordering of the system's decision-making agents that is consistent with the system's information structure. If such an association is possible, it leads to a partial order characterization of the corresponding information structure. In this paper we show that, in general, no such partial order characterization exists and we identify a sufficient (but not necessary) condition for existence. The condition has design implications that are important and intuitive and are discussed in the paper. Our results demonstrate that partial-order-based descriptions of concurrency, sometimes used in computer science, are not always correct.
\end{abstract}

Key words. Information structure, Concurrency, Nonsequential systems, Deadlock-freeness.

\section{Introduction-Motivation}

Most existing large-scale systems, including communication and computer networks, surveillance networks, economic systems, manufacturing systems, military systems, database systems, power systems, are informationally decentralized and nonsequential. ${ }^{1}$ These systems consist of several control stations (control agents, decision-makers) that have access to different information, communicate with one another and have to coordinate their strategies to optimize a common objective.

Two desirable features of any design of informationally decentralized nonsequential systems are deadlock-freeness (see Section 2, and [2]) and a high degree

\footnotetext{
* Date received: December 8, 1997. Date revised: May 30, 2000. This research was supported in part by NSF Grant NCR-9204419, AFOSR Grant F49620-96-1-0028, ARO Grant DAAH04-96-1-0377, ARPA Grant N00174-91-C-0116, and NSF Grant ECS-9979347.

$\dagger$ Department of Electrical Engineering and Computer Science, University of Michigan, Ann Arbor, Michigan 48109-2122, U.S.A. teneketzis@eecs.umich.edu.

$\ddagger$ Department of Electrical and Computer Engineering, University of Iowa, Iowa City, Iowa 522421595, U.S.A.msanders@icaen.uiowa.edu.

${ }^{1}$ Systems where the order of controlled actions depends on the events that occur in nature and/or on the system's control laws are called nonsequential.
} 
of concurrency. Informationally decentralized nonsequential systems are subject to deadlock (see Appendix A of [2], and [6]); deadlock-freeness allows the system to accomplish all of its desired tasks (functions). A high degree of concurrency allows the system to respond, in a timely fashion, to events critical to its operation.

Deadlock-freeness as well as the degree of concurrency of informationally decentralized nonsequential systems depend, through the systems' control laws (or protocols), on the systems' information structure, that is, on the information upon which each operation may base its action. When under some set of control laws no operation depends on any other's action, complete concurrency is possible (such a situation arises in static teams). Conversely, if under some set of laws all operations but the first in a series depend on the action of their predecessor in the series, no concurrency is possible. Between these extremes lie a host of alternatives. Under some control laws the dependencies may force operations to satisfy a fixed partial order. Under other protocols the partial order may depend on some exogenous input. Under still others the operations may be interdependent, causing deadlock.

The relationship between the information structure and deadlock-freeness has been investigated in [2], [3], and [10]. In this paper we explore the relationship between the information structure and concurrency. For that matter we investigate two variants of the following problem: Consider an informationally decentralized nonsequential system with a deadlock-free information structure $\mathscr{I}$. Characterize the precedence constraints imposed by this information structure on the system's controlled and uncontrolled inputs in terms of a function, say $\delta$, that has the following characteristic: It associates each realization of the inputs with the strongest partial ordering of the system's decision-making agents that is consistent with the information structure $\mathscr{I}$. By consistent we mean that for each realization of the system's controlled and uncontrolled inputs all total orders compatible with the information structure's precedence constraints are also compatible with the partial order generated by $\delta$ and vice versa.

The solution to the above problem, that is the function $\delta$, if it exists, clearly highlights the relationship between an informationally decentralized nonsequential system's information structure and its degree of concurency; furthermore, $\delta$ provides a partial order characterization of the system's information structure. We formulate two problems, (P1) and (P2), that address the existence of partial order characterizations of information structures. We show that, in general, no partial order characterizations of information structures exist. We identify for Problems (P1) and (P2) a sufficient condition for existence. This condition reveals, along any realization of the controlled and uncontrolled inputs, the information flow that results in a deadlock-free operation of the system. Furthermore, the same condition has design implications that are important and intuitive, and are discussed after the analysis of Problems (P1) and (P2).

To the best of our knowledge, the results of this paper present the first condition sufficient to ensure the existence of partial order characterizations of information structures. The question of partial order existence was first raised by Witsenhausen in [12]. More recently, the same question has been taken up in the concurrency literature (see [7]). The results of this paper resolve Witsenhausen's 
question and provide informational conditions to ensure that the "minimal causality" modeling discussed in [7] gives rise to unique partial order characterizations. The results of this paper may be surprising to some, as they demonstrate that partial-order-based descriptions of concurrency, sometimes used in computer science, are not always correct.

The paper is organized as follows: In Section 2 we present Witsenhausen's intrinsic model, a mathematical model that is appropriate for the investigation of the relationship between the information structure and concurrency. In Section 3 we discuss the properties that characterize deadlock-free information structures. In Section 4 we precisely formulate two problems addressing the relationship between information structure and concurrency. We analyze these problems in Section 5. In Section 6 we discuss possible extensions of the problems formulated in Section 4. We conclude our discussion in Section 7.

\section{The Intrinsic Model—Nonsequential Systems}

To investigate the relationship between the information structure and concurrency it is essential to model systems in a framework where every action can, in principle, affect the information upon which every other action is based. One such framework is provided by Witsenhausen's intrinsic model [10], [12].

Formally, the intrinsic model has three components:

1. An information structure $\mathscr{I}:=\left\{(\Omega, \mathscr{B}),\left(U^{k}, \mathscr{U}^{k}\right), \mathscr{J}^{k}: k=1, \ldots, N\right\}$ specifies the system's allowable decisions and distinguishable events.

(a) $N \in \mathbb{N}$ denotes the number of control actions to be taken.

(b) $(\Omega, \mathscr{B})$ denotes the measurable space from which a random input $\omega$ is drawn.

(c) $\left(U^{k}, \mathscr{U}^{k}\right)$ denotes the measurable space from which $u^{k}$, the $k$ th control action, is selected. ${ }^{2}$ It is assumed that card $\left(U^{k}\right)$ is greater than one, and that $\mathscr{U}^{k}$ contains the singletons of $U^{k}$. The product space containing the $N$-tuple of control actions, $u:=\left(u^{1}, u^{2}, \ldots, u^{N}\right)$, is denoted by $(U, \mathscr{U}):=$ $\left(\prod_{i=1}^{N} U^{i}, \otimes_{i=1}^{N} \mathscr{U}^{i}\right) .^{3}$

(d) The $\sigma$-field $\mathscr{J}^{k} \subset \mathscr{B} \otimes \mathscr{U}$ characterizes the maximal information that can be used to select the $k$ th control action.

2. A design constraint set $\Gamma_{C}$ constrains the set of admissible $N$-tuples of control laws, $\gamma:=\left(\gamma^{1}, \gamma^{2}, \ldots, \gamma^{N}\right)$, called designs, to a nonempty subset of $\Gamma:=$ $\prod_{i=1}^{N} \Gamma^{i}$, where $\Gamma^{k}, k=1,2, \ldots, N$, denotes the set of all $\mathscr{J}^{k} / \mathscr{U}^{k}$-measurable functions.

\footnotetext{
${ }^{2}$ From a game theoretic perspective (see [9]), the control actions can be viewed as being the actions of $N$ distinct decision-making agents (computers, devices, processes, etc.). Similarly, the random inputs can be viewed as a single action of nature (chance).

${ }^{3} \mathscr{X} \otimes \mathscr{Y}$ denotes the product $\sigma$-field of the $\sigma$-fields $\mathscr{X}$ and $\mathscr{Y}$; i.e., $\mathscr{X} \otimes \mathscr{Y}:=\sigma\left(\left[\pi_{X}\right]^{-1}(\mathscr{X}) \cup\right.$ $\left.\left[\pi_{Y}\right]^{-1}(\mathscr{Y})\right)$ is the smallest $\sigma$-field of $X \times Y$ for which the canonical projections $\pi_{X}\left(\pi_{X}(x, y)=x\right)$ and $\pi_{Y}\left(\pi_{Y}(x, y)=y\right)$ are both measurable (see [4] and [5]).
} 
3. A probability measure $P$ on $(\Omega, \mathscr{B})$ specifies the mixed (randomized) decision policy to be used by nature to select $\omega$.

Note that the intrinsic model does not exclude the possibility of an agent employing a mixed decision policy, or a policy that dictates that the agent not act. To model the mixed policy, randomizing devices can be included as factors in $(\Omega, \mathscr{B}, P)$, and the effects of the devices' outputs can be specified in $\mathscr{J}^{k}$. To model inaction, the agent can be allowed to make decisions that have no effect.

The intrinsic model, proposed by Witsenhausen [10], [12], has been used to model informationally decentralized nonsequential systems [2], [3], [10], [12] and to formulate and study decentralized nonsequential stochastic control problems [1]-[3], [8], [10], [12]. Within the framework of Witsenhausen's intrinsic model it was shown that informationally decentralized nonsequential systems can potentially perform better than sequential ones [2], that dynamic team problems are, in most cases, equivalent to static team problems [13], and a framework for the optimization of unconstrained decentralized nonsequential stochastic control problems has been proposed [1]. Even though informationally decentralized nonsequential systems can potentially perform better than sequential ones, they are subject to deadlock [2]. Furthermore, in informationally decentralized nonsequential systems it is not always possible to compute the performance associated with certain designs precisely [8]. Consequently, optimization problems associated with these systems are not always well-posed. That is why it is important to determine conditions on the information structure that (i) guarantee deadlock-free operation of informationally decentralized nonsequential systems and (ii) ensure that the performance associated with each design can be precisely quantified. The results of [10], [2], and [3] present properties of the information structure that are necessary and sufficient [2], [3], or sufficient [10] to ensure deadlock-freeness ${ }^{4}$ of nonsequential systems for all designs [10], [2], or for particular designs [3]. Furthermore, these properties of the information structure, called causality (Property C) [10] and causal implementability (Property CI) [2], ensure that the performance associated with each design can be precisely computed [10], [2]. Therefore, optimization of informationally decentralized nonsequential systems possessing Property $\mathrm{C}$ or Property $\mathrm{CI}$ is possible.

From the above discussion it is evident that it is desirable to design informationally decentralized nonsequential systems with an information structure that possesses the abovementioned properties. For this reason, in this paper we concentrate on information structures that possess Property C or Property CI. We find characterizations of these information structures that reveal the relationship between the information structure and concurrency and provide deep insight into fundamental issues associated with the performance of informationally decentralized nonsequential stochastic controlled systems such as: Who should know what and when? Who should communicate with whom and when?

\footnotetext{
${ }^{4}$ An information structure $\mathscr{I}$ is deadlock-free if for each $\gamma \in \Gamma$, and for every $\omega \in \Omega$, there exists an ordering of $\gamma$ 's $N$ control laws, say $\gamma^{s_{1}(\omega)}, \gamma^{s_{2}(\omega)}, \ldots, \gamma^{s_{N}(\omega)}$, such that no control action $u^{s_{n}(\omega)}, n=$ $1,2, \ldots, N$, depends on itself or the control actions that follow.
} 
Before we formulate the technical problems that we investigate in this paper we precisely define Properties $\mathrm{C}$ and $\mathrm{CI}$ as well as their design-dependent analogues, Properties $\mathrm{C}^{*}$ and $\mathrm{CI}^{*}$, respectively.

\section{Properties $\mathrm{CI}, \mathrm{C}, \mathrm{CI}^{*}$, and $\mathrm{C}^{*}$}

Before defining Properties $\mathrm{CI}, \mathrm{C}, \mathrm{CI}^{*}$, and $\mathrm{C}^{*}$ we establish some notation. We define by

$$
\mathscr{Z}:=\{1,2, \ldots, N\}
$$

the set of decision-making agents (see the specification of information structure, Section 2, and footnote 2). For all $k=1,2, \ldots, N$, we define $S_{k}$ to be the set of all $k$-agent orderings, i.e., all injections of $\{1,2, \ldots, k\}$ into $N$. We let

$$
T_{j}^{k}: S_{k} \rightarrow S_{j},
$$

$j=0,1,2, \ldots, N, k=j, j+1, \ldots, N$, denote a truncation map that returns the ordering $s$ of the first $j$ agents of a $k$-agent ordering; that is, $T_{j}^{k}$ restricts $s \in S_{k}$ to the domain $\{1,2, \ldots, j\}$ or to $\varnothing$ when $j=0$. For all $s \in S_{k}$ and $k=1,2, \ldots, N$, we define $\mathscr{P}_{s}$ to be the projection of $\Omega \times U$ onto $\Omega \times \prod_{i=1}^{k} U^{s_{i}}$; that is,

$$
\mathscr{P}_{s}(\omega, u):=\left(\omega, u^{s_{1}}, u^{s_{2}}, \ldots, u^{s_{k}}\right), \quad \mathscr{P}_{\varnothing}(\omega, u)=\omega .
$$

For any $H:=\left\{s_{1}, s_{2}, \ldots, s_{r}\right\} \subset \mathscr{Z}$ and any $(\omega, u) \in \Omega \times U$, we define

$$
\mathscr{P}_{H}(\omega, u):=\left(\omega, u^{s_{1}}, u^{s_{2}}, \ldots, u^{s_{r}}\right) .
$$

For any $\gamma \in \Gamma, \gamma:=\left(\gamma^{1}, \gamma^{2}, \ldots, \gamma^{N}\right)$, we denote by

$$
\mathscr{J}^{\gamma^{k}}:=\left\{\left[\gamma^{k}\right]^{-1}\left(u^{k}\right): u^{k} \in U^{k}\right\}, \quad k=1,2, \ldots, N,
$$

the information partition of agent $k$ induced by the control law $\gamma$. Finally, for any design $\gamma \in \Gamma$ we define the graph of $\gamma$ as

$$
G^{\gamma}:=\{(\omega, u): \gamma(\omega, u)=u\} .
$$

Definition 1 [2]. An information structure $\mathscr{I}$ possesses Property $C I$ when there exists at least one map $\psi: \Omega \times U \rightarrow S_{N}$ such that for all $k=1,2, \ldots, N$, and $(\omega, u) \in \Omega \times U$,

$$
\mathscr{J}^{s_{k}} \cap\left[\mathscr{P}_{T_{k-1}^{N}(s)}\right]^{-1}\left(\mathscr{P}_{T_{k-1}^{N}(s)}(\omega, u)\right) \subset\left\{\varnothing,\left[\mathscr{P}_{T_{k-1}^{N}(s)}\right]^{-1}\left(\mathscr{P}_{T_{k-1}^{N}(s)}(\omega, u)\right)\right\},
$$

when $s:=\left(s_{1}, s_{2}, \ldots, s_{N}\right)=\psi(\omega, u)$.

Property CI ensures that for all outcomes $(\omega, u) \in \Omega \times U$ there exists an order $s:=\left(s_{1}, s_{2}, \ldots, s_{N}\right)=\psi(\omega, u)$ such that for all $k=1,2, \ldots, N$, the $s_{k}$ th agent's information at the point $(\omega, u)$ depends only on the random input $\omega$ and the actions $u^{s_{1}}, u^{s_{2}}, \ldots, u^{s_{k-1}}$ of its predecessors in $s$.

Definition 2 [10]. An information structure $\mathscr{I}$ possesses Property $C$ when there exists at least one map $\psi: \Omega \times U \rightarrow S_{N}$ such that for all $s:=\left(s_{1}, s_{2}, \ldots, s_{k}\right) \in S_{k}$, 
$k=1,2, \ldots, N$,

$$
\mathscr{J}^{s_{k}} \cap\left[T_{k}^{N} \circ \psi\right]^{-1}(s) \subset \mathscr{F}\left(T_{k-1}^{k}(s)\right),
$$

where $\mathscr{F}(s)$ denotes the cylindrical extension of $\mathscr{B} \otimes\left(\bigotimes_{i=1}^{k} \mathscr{U}^{s_{i}}\right)$ to $\Omega \times U$ for all $s \in S_{k}, k=1,2, \ldots, N$.

As in Property CI, the order function $\psi$ maps every outcome in $\Omega \times U$ into an $N$-agent ordering. However, unlike Property CI, the order functions $\psi$ in Property $\mathrm{C}$ are constrained to be $(\mathscr{B} \otimes \mathscr{U})$-measurable (see [2]). Property $\mathrm{C}$ ensures that there exists an order function $\psi$, such that for all possible orderings $s \in S_{k}$, $k=1,2, \ldots, N$, the events that agent $s_{k}$ can distinguish (given that the ordering of the first $k$ agents, as determined by $\psi$, is $s$ ) are events that are induced by the random input $\omega$ and the actions of the $s_{k}$ agent's predecessors in $s$.

As pointed out in Section 2, Properties CI and C are design-independent, since they guarantee deadlock-free operation of informationally decentralized nonsequential systems for each design, and ensure that the performance associated with each design can be precisely quantified (see [2] and [10]). Properties CI and C have design-dependent analogues that are described by Properties $\mathrm{CI}^{*}$ and $\mathrm{C}^{*}$, respectively.

Definition 3 [3]. A design $\gamma$ possesses Property $C I^{*}$ when $\mathscr{P}_{\varnothing}\left(G^{\gamma}\right)=\Omega$, and there exists at least one map $\psi: G^{\gamma} \rightarrow S_{N}$ such that for all $k=1,2, \ldots, N$, and $(\omega, u) \in G^{\gamma}$,

$$
\mathscr{J}^{\gamma^{s_{k}}} \cap\left[\mathscr{P}_{T_{k-1}^{N}(s)}\right]^{-1}\left(\mathscr{P}_{T_{k-1}^{N}(s)}(\omega, u)\right) \subset\left\{\varnothing,\left[\mathscr{P}_{T_{k-1}^{N}(s)}\right]^{-1}\left(\mathscr{P}_{T_{k-1}^{N}(s)}(\omega, u)\right)\right\}
$$

when $s:=\left(s_{1}, s_{2}, \ldots, s_{N}\right)=\psi(\omega, u)$.

Property CI* ensures that for all outcomes $(\omega, u) \in G^{\gamma}$, the cylinder set

$$
\left[\mathscr{P}_{T_{k-1}^{N}(s)}\right]^{-1}\left(\mathscr{P}_{T_{k-1}^{N}}(\omega, u)\right)=\left[\mathscr{P}_{T_{k-1}^{N}(s)}\right]^{-1}\left(\omega, u^{s_{1}}, u^{s_{2}}, \ldots, u^{s_{k-1}}\right)
$$

induced on $\Omega \times U$ by $\omega$ and the action of the first $k-1$ agents in $s:=$ $\left(s_{1}, s_{2}, \ldots, s_{N}\right)=\psi(\omega, u)$ is a subset of all events containing $(\omega, u)$ in the information partition $\mathscr{J}^{\gamma^{s_{k}}}$ induced by the $s_{k}$ th agent's control law $\gamma^{s_{k}}$; that is, no event in $\mathscr{J}^{\gamma^{s_{k}}}$ containing $(\omega, u)$ depends on $u^{s_{k}}, u^{s_{k+1}}, \ldots, u^{s_{N}}$.

Definition 4 [3]. A design $\gamma \in \Gamma$ possesses Property $C^{*}$ when $\mathscr{P}_{\varnothing}\left(G^{\gamma}\right)=\Omega$ and there exists at least one map $\psi: G^{\gamma} \rightarrow S_{N}$ such that for all $s:=\left(s_{1}, s_{2}, \ldots, s_{k}\right) \in S_{k}$ and $k=1,2, \ldots, N$,

$$
\begin{aligned}
\mathscr{J}^{\gamma^{s_{k}}} & \cap\left[\mathscr{P}_{T_{k-1}^{k}(s)}\right]^{-1}\left(\mathscr{P}_{T_{k-1}^{k}(s)}\left(\left[T_{k}^{N} \cdot \psi\right]^{-1}(s)\right)\right) \\
& \subset \mathscr{F}\left(T_{k-1}^{k}(s)\right) \cap\left[\mathscr{P}_{T_{k-1}^{k}(s)}\right]^{-1}\left(\mathscr{P}_{T_{k-1}^{k}(s)}\left(G^{\gamma}\right)\right) .
\end{aligned}
$$

Property $C^{*}$ ensures that there exists an order function $\psi$ such that for all possible orderings $s \in S_{k}, k=1,2, \ldots, N$, the events agent $s_{k}$ can distinguish under $\gamma$, given that the ordering of the first $k$ agents, as determined by $\psi$, is $s$, are events 
that can be induced in $\left[P_{T_{k-1}^{k}(s)}\right]^{-1}\left(P_{T_{k-1}^{k}(s)}\left(G^{\gamma}\right)\right)$ by the random input $\omega$ and the actions of the $s_{k}$ th agent's predecesors in $s$.

It was shown in [2] that Property $\mathrm{C}$ always implies Property $\mathrm{CI}$; this means that all order functions $\psi$ for which the information structure $\mathscr{I}$ possesses Property $\mathrm{C}$ are functions for which $\mathscr{I}$ possesses Property CI. In general there are order functions $\psi$ for which $\mathscr{I}$ possesses Property CI but not Property C (see [2]). It was proven in [2] that Properties $\mathrm{C}$ and $\mathrm{CI}$ are equivalent when the information structure $\mathscr{I}$ is sequential, ${ }^{5}$ or when $U^{k}, k=1,2, \ldots, N$, are countable sets and $\mathscr{B}$ contains the singletons of $\Omega$. Relations similar to the above hold between Properties $\mathrm{CI}^{*}$ and $\mathrm{C}^{*}$ (see Section 3.4 of [3]). Since Properties C and CI are not equivalent in general, the relationship between concurrency and information structures that possess (i) Property CI and (ii) Property C, has to be investigated separately. Similarly, a design's degree of concurrency has to be investigated separately for designs that possess (i) Property CI* and (ii) Property C*.

For an information structure $\mathscr{I}$ possessing Property CI (Property C) the set $\Psi$ $\left(\Psi^{\prime}\right)$ of all total order functions $\psi: \Omega \times U \rightarrow S_{N}$ for which Property CI (C) holds may be a large one. For example, as Witsenhausen points out in [12], when the system is a static team, $\Psi^{\prime}$ contains, in particular, all $\mathscr{F}(\varnothing)$-measurable functions, where $\mathscr{F}(\varnothing)$ denotes the cylindrical extension of $\mathscr{B}$ to $\Omega \times U$. Similar results hold for designs that possess Property $\mathrm{CI}^{*}$ or Property $\mathrm{C}^{*}$. In this paper we determine a condition (see Assumption A1 in Section 5.1) under which we can characterize the set $\Psi\left(\Psi^{\prime}\right)$ in terms of a function $\delta\left(\delta^{\prime}\right)$ from $\Omega \times U$ to partial orders on the set of agents $\mathscr{Z}$. That is, $\Psi\left(\Psi^{\prime}\right)$ is precisely the set of total order functions $\mu: \Omega \times U \rightarrow S_{N}$ that are compatible with $\delta\left(\delta^{\prime}\right)$. The function $\delta\left(\delta^{\prime}\right)$ provides a partial-order characterization of the (deadlock-free) information structure $\mathscr{I}$, and describes the relationship between $\mathscr{I}$ and the degree of concurrency of the corresponding informationally decentralized nonsequential system. Furthermore, $\delta\left(\delta^{\prime}\right)$ reveals, along each realization of controlled and uncontrolled inputs, the information flow that results in a deadlock-free operation of the system. In the next section we formulate precisely the problems of characterizing the sets $\Psi$ and $\Psi^{\prime}$ in terms of the functions of $\delta$ and $\delta^{\prime}$, respectively, and then proceed to analyze them. The design-dependent analogues of these problems are presented and discussed in Section 6.

\section{Problem Formulation}

We consider an informationally decentralized nonsequential system modeled by the intrinsic model with an information structure $\mathscr{I}=\left\{(\Omega, \mathscr{B}),\left(U^{k}, \mathscr{U}^{k}\right), \mathscr{J}^{k}\right.$, $k=1,2, \ldots, N\}$ and $\Gamma_{C}=\Gamma$. We assume that $\mathscr{I}$ possesses Property CI (Property C). Let $\Psi\left(\Psi^{\prime}\right)$ be the set of total order functions $\psi: \Omega \times U \rightarrow S_{N}$ for which $\mathscr{I}$ possesses Property CI $(C)$. We wish to find a simple characterization of $\Psi\left(\Psi^{\prime}\right)$ in

\footnotetext{
${ }^{5}$ An information structure $\mathscr{I}$ is said to be sequential when Property CI holds for some constant order function.
} 
terms of a function $\delta\left(\delta^{\prime}\right)$ from $\Omega \times U$ to partial orders on the set $\mathscr{Z}$ of agents. More specifically, we are concerned with the following problems:

(P1) Under what conditions is there a function $\delta$ from $\Omega \times U$ to partial orders on $\mathscr{Z}$ such that $\Psi=M$, where $M$ is the set of total order functions $\mu: \Omega$ $\times U \rightarrow S_{N}$ that are compatible with $\delta$ ?

(P2) Under what conditions is there a function $\delta^{\prime}$ from $\Omega \times U$ to partial orders on $\mathscr{Z}$ such that $\Psi^{\prime}=M^{\prime}$, where $M^{\prime}$ is the set of total order functions $\mu^{\prime}: \Omega \times U \rightarrow S_{N}$ that are compatible with $\delta^{\prime}$ and have the following property: for all $s \in S_{k}$ and all $k=1,2, \ldots, N,\left[T_{k}^{N} \cdot \mu^{\prime}\right]^{-1}(s) \in$ $\mathscr{F}\left(T_{k-1}^{k}(s)\right)$ ?

If there is a function $\delta\left(\delta^{\prime}\right)$ from $\Omega \times U$ to partial orders on the set of agents $\mathscr{Z}$ that solves Problem (P1) (Problem (P2)), then, along each intrinsic event $(\omega, u) \in$ $\Omega \times U, \delta(\omega, u)\left(\delta^{\prime}(\omega, u)\right)$ is the strongest partial order compatible with all $\psi(\omega, u), \psi \in \Psi$ (respectively, all $\left.\psi^{\prime}(\omega, u), \psi^{\prime} \in \Psi^{\prime}\right)$.

We proceed with the analysis of Problems (P1) and (P2).

\section{Analysis}

\subsection{The Main Condition}

We make the following assumption:

(A1) For each intrinsic event $(\omega, u)$ and for each agent $s_{m} \in \mathscr{Z}$ there is a unique smallest cardinality set $\Lambda^{s_{m}}(\omega, u) \subset \mathscr{Z}$ with the following properties:

$$
\begin{gathered}
\mathscr{J}^{s_{m}} \cap\left[\mathscr{P}_{\Lambda^{s m}(\omega, u)}\right]^{-1}\left(\mathscr{P}_{\Lambda^{s m}(\omega, u)}(\omega, u)\right) \\
\subset\left\{\varnothing,\left[\mathscr{P}_{\Lambda^{s m}(\omega, u)}\right]^{-1}\left(\mathscr{P}_{\Lambda^{s m}(\omega, u)}(\omega, u)\right)\right\} ; \\
\mathscr{J}^{s_{m}} \cap\left[\mathscr{P}_{\mathscr{L}}\right]^{-1}\left(\mathscr{P}_{\mathscr{L}}(\omega, u)\right) \not \subset\left\{\varnothing,\left[\mathscr{P}_{\mathscr{L}}\right]^{-1}\left(\mathscr{P}_{\mathscr{L}}(\omega, u)\right)\right\}
\end{gathered}
$$

for any set $\mathscr{L} \subset \mathscr{Z}$ such that $\Lambda^{s_{m}}(\omega, u) \not \subset \mathscr{L}$.

Equation (5.1) in (A1) is equivalent to the following condition: $\forall A \in \mathscr{J}^{s_{m}}$ such that $(\omega, u) \in A$,

$$
A \cap\left[\mathscr{P}_{\Lambda^{s_{m}}(\omega, u)}\right]^{-1}\left(\mathscr{P}_{\Lambda^{s_{m}}(\omega, u)}(\omega, u)\right)=\left[\mathscr{P}_{\Lambda^{s_{m}}(\omega, u)}\right]^{-1}\left(\mathscr{P}_{\Lambda^{s_{m}}(\omega, u)}(\omega, u)\right) .
$$

The meaning of Condition (5.1) (and its equivalent Condition (5.3)) is the following: Agent $s_{m}$ 's information at the point $(\omega, u)$ depends on the random input $\omega$ and the actions of agents in $\Lambda^{s_{m}}(\omega, u)$. The set $\Lambda^{s_{m}}(\omega, u)$ specifies the smallest subset of agents in $\mathscr{Z}$ that must communicate directly or indirectly with agent $s_{m}$ along $(\omega, u)$ so that $s_{m}$ can act. Consequently, along $(\omega, u)$ agent $s_{m}$ cannot act until $\omega$ occurs and all agents in $\Lambda^{s_{m}}(\omega, u)$ have acted; this is precisely expressed by Condition (5.2). The sets $\Lambda^{s_{m}}(\omega, u)$ reveal, along any intrinsic event $(\omega, u)$, the information flow that results in a deadlock-free operation of an informationally decentralized nonsequential system. That is, they specify the agents that must communicate with one another along any intrinsic event so that deadlock-freeness 
is achieved. In fact, for nonsequential systems the sets $\Lambda^{s_{m}}(\omega, u)$ play a role similar to that of the last component of the triple (Y, U, L) that defines the "field-basis" [11, p. 1559] in sequential stochastic controlled systems.

We present here one instance where Assumption (A1) is satisfied. Consider the following thought experiment. Take an intrinsic event $(\omega, u)$ and proceed as follows: First determine all agents $j \in \mathscr{Z}$ such that for all $A \in \mathscr{J}^{j}$ that contain $(\omega, u)$ the following condition is satisfied:

$$
A \cap\left[\mathscr{P}_{\varnothing}\right]^{-1}\left(\mathscr{P}_{\varnothing}(\omega, u)\right)=\left[\mathscr{P}_{\varnothing}\right]^{-1}\left(\mathscr{P}_{\varnothing}(\omega, u)\right) .
$$

Record all these agents. Call this set of agents $Z_{1}(\omega, u)$. This is the largest set of agents that can act based only on the occurrence of $\omega$. Let card $\left(Z_{1}(\omega, u)\right)=k$. Because $\mathscr{I}$ possesses Property CI, $k \geq 1$. Next, consider $\left(\omega, u^{s_{1}}, u^{s_{2}}, \ldots, u^{s_{k}}\right)$, where $Z_{1}(\omega, u):=\left(s_{1}, s_{2}, \ldots, s_{k}\right)$. Determine all agents $\ell \in\{1,2, \ldots, N\} \backslash Z_{1}(\omega, u)$ such that for all $A \in \mathscr{J}^{\ell}$ that contain $(\omega, u)$ the following condition is satisfied:

$$
A \cap\left[\mathscr{P}_{Z_{1}(\omega, u)}\right]^{-1}\left(\mathscr{P}_{Z_{1}(\omega, u)}(\omega, u)\right)=\left[\mathscr{P}_{Z_{1}(\omega, u)}\right]^{-1}\left(\mathscr{P}_{Z_{1}(\omega, u)}(\omega, u)\right) .
$$

Record all these agents, and call this set of agents $Z_{2}(\omega, u)$. This is the largest set of agents that can act based on $\omega$ and $\left(u^{s_{1}}, u^{s_{2}}, \ldots, u^{s_{k}}\right)$. Again, because $\mathscr{I}$ possesses Property CI, card $\left(Z_{2}(\omega, u)\right) \geq 1$. Continue this process along $(\omega, u)$ until all agents are accounted for. Repeat the same process as above for all intrinsic events $(\omega, u) \in \Omega \times U$. This completes the thought experiment.

Consider now the situation where the following is true: For every intrinsic event $(\omega, u)$ and every agent $s_{k}, k=1,2, \ldots, N$, if $s_{k} \in Z_{n}(\omega, u)$, then

$$
\Lambda^{s_{k}}(\omega, u)=\bigcup_{\ell=1}^{n-1} Z_{\ell}(\omega, u) .
$$

Then Assumption (A1) is satisfied. That is, Assumption (A1) is satisfied if along every intrinsic event $(\omega, u)$ the information of any agent depends on the actions of all the agents that act in the preceding stages of the experiment described above.

It is worth noting that, for any design $\gamma \in \Gamma$ and any $\omega \in \Omega$, the thought experiment described above specifies the partial ordering of agent actions that a passive observer would record (given $\omega$ ) if $\gamma$ were implemented in a "maximally" concurrent fashion.

We proceed to analyze Problems (P1) and (P2) under Assumption (A1).

\subsection{Analysis of Problem (P1)}

The following theorem, that is the main result of Section 5.2, shows that Problem (P1) has a solution if Assumption (A1) is satisfied.

Theorem 1. Consider an informationally decentralized nonsequential system modeled by the intrinsic model. Assume that the information structure II possesses Property $C I$, and let $\Psi$ be the set of total order functions $\psi: \Omega \times U \rightarrow S_{N}$ for which $\mathscr{I}$ possesses Property CI. Then there exists a function $\delta$ from $\Omega \times U$ to partial orders on the set $\mathscr{Z}$ of agents such that $\Psi=M$, where $M$ is the set of total order functions $\mu: \Omega \times U \rightarrow S_{N}$ that are compatible with $\delta$, if (A1) holds. 
Proof. Consider, for each intrinsic event $(\omega, u)$ and for each agent $s_{m} \in \mathscr{Z}$, the set $\Lambda^{s_{m}}(\omega, u)$ (defined in (A1)). For each intrinsic event $(\omega, u)$ define a precedence relation $\delta(\omega, u)$ on the set $\mathscr{Z}$ of agents as follows: $s_{i} \delta(\omega, u) s_{m}$ ( $s_{i}$ is a precedent of $s_{m}$ along $\left.(\omega, u)\right)$, if $s_{i}$ satisfies one of the following two conditions:

(C1) $s_{i} \in \Lambda^{s_{m}}(\omega, u) \cup\left\{s_{m}\right\}$;

(C2) there exists at least one sequence of agents $s_{\alpha_{1}}, s_{\alpha_{2}}, \ldots, s_{\alpha_{\ell}}$ such that $s_{i} \in \Lambda^{s_{\alpha_{1}}}(\omega, u), s_{\alpha_{1}} \in \Lambda^{s_{\alpha_{2}}}(\omega, u), s_{\alpha_{2}} \in \Lambda^{s_{\alpha_{3}}}(\omega, u), \ldots, s_{\alpha_{\ell}} \in \Lambda^{s_{m}}(\omega, u)$.

The precedence relation defined above has the following characteristics:

(i) If $s_{k} \delta(\omega, u) s_{\ell}$ and $s_{\ell} \delta(\omega, u) s_{k}$, then $s_{k}=s_{\ell}$ because the information structure $\mathscr{I}$ possesses Property CI.

(ii) If $s_{k} \delta(\omega, u) s_{\ell}$ and $s_{\ell} \delta(\omega, u) s_{m}$, then $s_{k} \delta(\omega, u) s_{m}$.

Consequently, for all $(\omega, u) \in \Omega \times U, \delta(\omega, u)$ is antisymmetric and transitive. Furthermore, because of $(\mathrm{C} 1)$, it is reflexive. Hence, for every $(\omega, u), \delta(\omega, u)$ is a partial order on the set $\mathscr{Z}$ of agents.

We now prove that all total order functions $\psi$ compatible with $\delta$ are such that $\mathscr{I}$ possesses Property CI. Consider any total order function $\psi$,

$$
\psi: \Omega \times U \rightarrow S_{N}
$$

that is compatible with $\delta$, and consider any intrinsic event $(\omega, u)$. Let $\psi(\omega, u)=$ $\left(s_{1}, s_{2}, \ldots, s_{N}\right):=s$, and consider any agent $s_{k}$. We want to prove that

$$
\mathscr{J}^{s_{k}} \cap\left[\mathscr{P}_{T_{k-1}^{N}(s)}\right]^{-1}\left(\mathscr{P}_{T_{k-1}^{N}(s)}(\omega, u)\right) \subset\left\{\varnothing,\left[\mathscr{P}_{T_{k-1}^{N}(s)}\right]^{-1}\left(\mathscr{P}_{T_{k-1}^{N}(s)}(\omega, u)\right)\right\},
$$

or equivalently that $\forall A \in \mathscr{J}^{s_{k}}$ such that $(\omega, u) \in A$,

$$
A \cap\left[\mathscr{P}_{T_{k-1}^{N}(s)}\right]^{-1}\left(\mathscr{P}_{T_{k-1}^{N}(s)}(\omega, u)\right)=\left[\mathscr{P}_{T_{k-1}^{N}(s)}\right]^{-1}\left(\mathscr{P}_{T_{k-1}^{N}(s)}(\omega, u)\right) .
$$

Since $\psi$ is compatible with $\delta$ it follows, from the definition of $\delta$, that $\left\{s_{1}, s_{2}, \ldots, s_{k-1}\right\} \supset \Lambda^{s_{k}}(\omega, u)$. Therefore,

$$
\left[\mathscr{P}_{T_{k-1}^{N}(s)}\right]^{-1}\left(\mathscr{P}_{T_{k-1}^{N}(s)}(\omega, u)\right) \subset\left[\mathscr{P}_{\Lambda^{s_{k}}(\omega, u)}\right]^{-1}\left(\mathscr{P}_{\Lambda^{s_{k}}(\omega, u)}(\omega, u)\right) .
$$

Combining (5.9) with (5.3) we conclude that $\forall A \in \mathscr{J}^{s_{k}}$ such that $(\omega, u) \in A$,

$$
A \cap\left[\mathscr{P}_{T_{k-1}^{N}(s)}\right]^{-1}\left(\mathscr{P}_{T_{k-1}^{N}(s)}(\omega, u)\right)=\left[\mathscr{P}_{T_{k-1}^{N}(s)}\right]^{-1}\left(\mathscr{P}_{T_{k-1}^{N}(s)}(\omega, u)\right) .
$$

Since (5.10) is true for any intrinsic event $(\omega, u) \in \Omega \times U$ and for any agent $s_{k}$, it follows that all total order functions $\psi$ compatible with $\delta$ are such that $\mathscr{I}$ possesses Property CI. Hence,

$$
M \subset \Psi .
$$

Next we prove that all total order functions $\psi$ such that $\mathscr{I}$ possesses Property $\mathrm{CI}$ are compatible with $\delta$. Suppose there is a total order function $\psi$ such that $\mathscr{I}$ possesses Property $\mathrm{CI}$ and $\psi$ is not compatible with $\delta$. Then there exists at least one intrinsic event $(\omega, u)$ such that the total order $\psi(\omega, u)=\left(s_{1}, s_{2}, \ldots, s_{N}\right):=s$ cannot be obtained by $\delta(\omega, u)$. This means that there exist agents $s_{k}, s_{\ell}$ such that $s_{k}$ 's action precedes $s_{\ell}$ 's action according to $\psi(\omega, u)=s$ and $s_{\ell} \delta(\omega, u) s_{k}$. Since $s_{\ell} \delta(\omega, u) s_{k}$ it follows that either $s_{\ell} \in \Lambda^{s_{k}}(\omega, u)$ or there exists a sequence of agents 
$s_{i_{1}}, s_{i_{2}}, \ldots, s_{i_{m}}$ such that $s_{\ell} \in \Lambda^{s_{i_{1}}}(\omega, u), s_{i_{1}} \in \Lambda^{s_{i_{2}}}(\omega, u), \ldots, s_{i_{m}} \in \Lambda^{s_{k}}(\omega, u)$. Consider first the case where $s_{\ell} \in \Lambda^{s_{k}}(\omega, u)$. In this case, since, according to $\psi, s_{\ell} \notin T_{k-1}^{N}(s)$, it follows from (5.2) that

$$
\mathscr{J}^{s_{k}} \cap\left[\mathscr{P}_{T_{k-1}^{N}(s)}\right]^{-1}\left(\mathscr{P}_{T_{k-1}^{N}(s)}(\omega, u)\right) \not \subset\left\{\varnothing,\left[\mathscr{P}_{T_{k-1}^{N}(s)}\right]^{-1}\left(\mathscr{P}_{T_{k-1}^{N}(s)}(\omega, u)\right)\right\} .
$$

However, (5.12) contradicts the fact that $\psi$ is such that $\mathscr{I}$ possesses Property CI (see relation (3.7)). Consequently, it is not possible to have $s_{\ell} \in \Lambda^{s_{k}}(\omega, u)$.

Suppose next that there exist agents $s_{i_{1}}, s_{i_{2}}, \ldots, s_{i_{m}}$ such that $s_{\ell} \in \Lambda^{s_{i_{1}}}(\omega, u)$, $s_{i_{1}} \in \Lambda^{s_{i_{2}}}(\omega, u), \ldots, s_{i_{m}} \in \Lambda^{s_{k}}(\omega, u)$. Since $\psi$ is such that $\mathscr{I}$ possesses Property CI and $s_{\ell} \in \Lambda^{s_{i_{1}}}(\omega, u)$, it follows from (5.1) and (5.2) that, according to $\psi, s_{i_{1}}$ must act after $s_{\ell}$ along $(\omega, u)$. By successive applications of the same argument we find that, according to $\psi, s_{i_{2}}, s_{i_{3}}, \ldots, s_{i_{m}}$ must act after $s_{\ell}$ along $(\omega, u)$. Consequently, $s_{i_{m}} \notin T_{k-1}^{N}(s)$ because $s_{k}$ 's action precedes $s_{\ell}$ 's action according to $\psi(\omega, u)$. Hence $\Lambda^{s_{k}}(\omega, u) \not \subset T_{k-1}^{N}(s)$, and (5.2) implies that

$$
\mathscr{J}^{s_{k}} \cap\left[\mathscr{P}_{T_{k-1}^{N}(s)}\right]^{-1}\left(\mathscr{P}_{T_{k-1}^{N}(s)}(\omega, u)\right) \not \subset\left\{\varnothing,\left[\mathscr{P}_{T_{k-1}^{N}(s)}\right]^{-1}\left(\mathscr{P}_{T_{k-1}^{N}(s)}(\omega, u)\right)\right\} .
$$

However, (5.13) contradicts the fact that $\psi$ is such that $\mathscr{I}$ possesses Property CI (see relation (3.7)). Therefore, it is impossible to have a total order function $\psi$ such that $\mathscr{I}$ possesses Property CI and an intrinsic event $(\omega, u)$ such that $\psi(\omega, u)=\left(s_{1}, s_{2}, \ldots, s_{k}, \ldots, s_{\ell}, \ldots, s_{n}\right)$ and $s_{\ell} \delta(\omega, u) s_{k}$ for some $s_{k}, s_{\ell}$.

Consequently, there does not exist any total order function $\psi$ for which $\mathscr{I}$ possesses Property CI and that is incompatible with $\delta$. Hence,

$$
\Psi \subset M .
$$

Relations (5.11) and (5.14) imply that $\Psi=M$.

The proof of Theorem 1 is now complete.

Next we illustrate that if (A1) is not satisfied, Problem (P1) does not, in general, have a solution.

Example 1. Consider the following information structure $\mathscr{I}$ :

$$
\begin{aligned}
N & =3, \\
\Omega & =U^{1}=U^{2}=U^{3}=\{0,1\}, \\
\mathscr{B} & =\mathscr{U}^{1}=\mathscr{U}^{2}=\mathscr{U}^{3}=\{\varnothing,\{0\},\{1\},\{0,1\}\}, \\
\mathscr{J}^{1} & =\{\varnothing,\{(\omega, u): \omega=0\},\{(\omega, u): \omega=1\}, \Omega \times U\}, \\
\mathscr{J}^{2} & =\{\varnothing,\{(\omega, u): \omega=0\},\{(\omega, u): \omega=1\}, \Omega \times U\}, \\
\mathscr{J}^{3} & =\left\{\varnothing,\left\{(\omega, u): u^{1} u^{2}=0\right\},\left\{(\omega, u): u^{1} u^{2}=1\right\}, \Omega \times U\right\} .
\end{aligned}
$$

Along any of the intrinsic events $\left(\omega, 0,0, u^{3}\right), \omega \in\{0,1\}, u^{3} \in\{0,1\}$, there is not a unique smallest cardinality set $\Lambda^{3}\left(\omega, 0,0, u^{3}\right)$ satisfying (5.1) and (5.2). We have

$$
\Lambda^{3}\left(\omega, 0,0, u^{3}\right)=\{1\} \quad \text { and } \quad \Lambda^{3}\left(\omega, 0,0, u^{3}\right)=\{2\}
$$


satisfying (5.1) and (5.2). Thus, Assumption (A1) is not satisfied. Suppose there is a function $\delta$ from $\Omega \times U$ to partial orders on the set $\{1,2,3\}$ of agents that solves Problem (P1). Consider the total order functions $\psi_{i}: \Omega \times U \rightarrow S_{3}, i=1,2$, defined by

$$
\psi_{1}(\omega, u)= \begin{cases}(2,3,1), & \text { if } u^{1}=u^{2}=0 \\ (2,1,3), & \text { otherwise }\end{cases}
$$

and

$$
\psi_{2}(\omega, u)= \begin{cases}(1,3,2), & \text { if } u^{1}=u^{2}=0 \\ (2,1,3), & \text { otherwise. }\end{cases}
$$

The functions $\psi_{1}$ and $\psi_{2}$ are such that (3.7) is satisfied for all $(\omega, u)$ and for all agents. Therefore, $\psi_{1}$ and $\psi_{2}$ are order functions such that $\mathscr{I}$ possesses Property CI. Since by assumption $\delta$ solves Problem (P1), $\psi_{1}$ and $\psi_{2}$ are compatible with $\delta$. Then the total order function $\psi_{3}: \Omega \times U \rightarrow S_{3}$, defined by

$$
\psi_{3}(\omega, u)= \begin{cases}(3,2,1), & \text { if } u^{1}=u^{2}=0 \\ (2,1,3), & \text { otherwise, }\end{cases}
$$

is compatible with $\delta$. Hence, $\psi_{3}$ is such that $\mathscr{I}$ possesses Property CI. However, at $(0,0,0,0)$, where $\psi_{3}(0,0,0,0)=(3,2,1),(3.7)$ is not satisfied because

$$
\mathscr{J}^{3} \cap\left(\{0\} \times U^{1} \times U^{2} \times U^{3}\right) \not \subset\left\{\varnothing,\{0\} \times U^{1} \times U^{2} \times U^{3}\right\} .
$$

Thus, under the assumption that there is a function $\delta$ from $\Omega \times U$ to partial orders on the set $\{1,2,3\}$ of agents that solves Problem (P1), a contradiction is reached. Consequently, there is no function $\delta$ from $\Omega \times U$ to partial orders on $\{1,2,3\}$ that solves Problem $(\mathrm{P} 1)$.

The validity of Assumption (A1) is sufficient to ensure that Problem (P1) has a solution, but it is not necessary as the following example shows.

Example 2. Consider the following information structure $\mathscr{I}$ :

$$
\begin{aligned}
N & =3, \\
\Omega & =U^{1}=U^{2}=U^{3}=\{0,1\}, \\
\mathscr{B} & =\mathscr{U}^{1}=\mathscr{U}^{2}=\mathscr{U}^{3}=\{\varnothing,\{0\},\{1\},\{0,1\}\}, \\
\mathscr{J}^{1} & =\{\varnothing,\{(\omega, u): \omega=0\},\{(\omega, u): \omega=1\}, \Omega \times U\}, \\
\mathscr{J}^{2} & =\left\{\varnothing,\left\{(\omega, u): u^{1}=0\right\},\left\{(\omega, u): u^{1}=1\right\}, \Omega \times U\right\}, \\
\mathscr{J}^{3} & =\left\{\varnothing,\left\{(\omega, u): u^{1} u^{2}=0\right\},\left\{(\omega, u): u^{1} u^{2}=1\right\}, \Omega \times U\right\} .
\end{aligned}
$$

Along any of the intrinsic events $\left(\omega, 0,0, u^{3}\right), \omega \in\{0,1\}, u^{3} \in\{0,1\}$, there is not a unique smallest cardinality set $\Lambda^{3}\left(\omega, 0,0, u^{3}\right)$ satisfying (5.1) and (5.2). We have

$$
\Lambda^{3}\left(\omega, 0,0, u^{3}\right)=\{1\} \text { and } \Lambda^{3}\left(\omega, 0,0, u^{3}\right)=\{2\}
$$

satisfying (5.1) and (5.2). Hence, Assumption (A1) is not satisfied. Consider the 
following function $\delta$, constructed as in the proof of Theorem 1:

At $\left(\omega, 0, u^{2}, u^{3}\right)$,

$$
1 \delta\left(\omega, 0, u^{2}, u^{3}\right) 2
$$

and

$$
1 \delta\left(\omega, 0, u^{2}, u^{3}\right) 3 .
$$

$\operatorname{At}\left(\omega, 1, u^{2}, u^{3}\right)$,

$$
1 \delta\left(\omega, 1, u^{2}, u^{3}\right) 2
$$

and

$$
2 \delta\left(\omega, 1, u^{2}, u^{3}\right) 3 .
$$

There are $2^{8}$ total order functions $\mu: \Omega \times U \rightarrow S_{3}$ that are compatible with the function $\delta$ defined by (5.33)-(5.36). These are precisely the total order functions for which $\mathscr{I}$ possesses Property CI. Thus, $M=\Psi$, and the function $\delta$ from $\Omega \times U$ to partial orders on $\{1,2,3\}$, defined by (5.33)-(5.36), solves Problem (P1) even though (A1) is not satisfied.

\subsection{Analysis of Problem (P2)}

The following theorem, that is the main result of Section 5.3, shows that Problem (P2) has a solution if Assumption (A1) is satisfied.

Theorem 2. Consider an informationally decentralized nonsequential system modeled by the intrinsic model. Suppose that the information structure $\mathscr{I}$ possesses Property $C$, and let $\Psi^{\prime}$ be the set of total order functions $\psi^{\prime}: \Omega \times U \rightarrow S_{N}$ for which $\mathscr{I}$ possesses Property $C$. Then there exists a function $\delta^{\prime}$ from $\Omega \times U$ to partial orders on the set $\mathscr{Z}$ of agents such that $\Psi^{\prime}=M^{\prime}$ if Assumption (A1) holds. $M^{\prime}$ is defined to be the set of total order functions $\mu^{\prime}: \Omega \times U \rightarrow S_{N}$ that are compatible with $\delta^{\prime}$ and have the following property: for all $s \in S_{k}$ and all $k=1,2, \ldots, N$, $\left[T_{k}^{N} \cdot \mu^{\prime}\right]^{-1}(s) \in \mathscr{F}\left(T_{k-1}^{k}(s)\right)$.

Proof. Take $\delta^{\prime}=\delta$, where $\delta$ is the function from $\Omega \times U$ to partial orders on $\mathscr{Z}$ constructed in the proof of Theorem 1. We prove that $\delta$ solves Problem (P2).

(i) Consider any $\psi^{\prime} \in \Psi^{\prime}$. Since Property C implies Property CI (see Corollary 1 of [2]), $\psi^{\prime} \in \Psi$ (where $\Psi$ is the set of total order functions from $\Omega \times U$ to $S_{N}$ such that $\mathscr{I}$ possesses Property CI). From Theorem 1 it follows that $\psi^{\prime}$ is compatible with $\delta$. Furthermore, since $\psi^{\prime} \in \Psi^{\prime}$, Lemma 5 of [10] implies that for all $s \in S_{k}$ and all $k=1,2, \ldots, N,\left(T_{k}^{N} \cdot \psi^{\prime}\right)^{-1}(s) \in \mathscr{\mathscr { F }}\left(T_{k-1}^{k}(s)\right)$. Consequently,

$$
\Psi^{\prime} \subset M^{\prime} .
$$

(ii) Suppose there is $\mu^{\prime} \in M^{\prime}$ such that $\mu^{\prime} \notin \Psi^{\prime}$. Then there exists at least one intrinsic event $(\omega, u)$, some $k=1,2, \ldots, N$, and $A \in \mathscr{J}^{s_{k}}$ such that

$$
A \cap\left[T_{k}^{N} \cdot \mu^{\prime}\right]^{-1}(s) \notin \mathscr{F}\left(T_{k-1}^{k}(s)\right),
$$

where

$$
s:=\left(s_{1}, s_{2}, \ldots, s_{k}\right)=T_{k}^{N}\left(\mu^{\prime}(\omega, u)\right) \in S_{k} .
$$


Since by assumption $\left[T_{k}^{N} \cdot \mu^{\prime}\right]^{-1}(s) \in \mathscr{F}\left(T_{k-1}^{k}(s)\right),(5.38)$ implies that

$$
A \notin \mathscr{F}\left(T_{k-1}^{k}(s)\right) \text {. }
$$

Hence, along $(\omega, u)$ agent $s_{k}$ 's information depends on the action of at least one of its successors in $\mu^{\prime}(\omega, u)$, say agent $s_{\ell}$. This means that $s_{\ell} \delta(\omega, u) s_{k}$ (by the construction of $\delta$ ) which in turn implies that $\mu^{\prime}$ is not compatible with $\delta$. Thus, a contradiction is reached (because by assumption $\mu^{\prime} \in M^{\prime}$ ). Consequently, every $\mu^{\prime} \in M^{\prime}$ is such that $\mu^{\prime} \in \Psi^{\prime}$. Hence

$$
M^{\prime} \subset \Psi^{\prime}
$$

Combining (5.37) and (5.41) we obtain $M^{\prime}=\Psi^{\prime}$. The proof of Theorem 2 is now complete.

Remarks. 1. In the information structures considered in Examples 1 and $2, \Omega$ and $U^{k}, k=1,2,3$, are finite sets and $\mathscr{B}$ contains the singletons of $\Omega$. Therefore, by Theorem 4 in [2], Properties $\mathrm{C}$ and $\mathrm{CI}$ are equivalent. Consequently, Example 1 illustrates that if (A1) is not satisfied, Problem (P2) does not, in general, have a solution. Furthermore, Example 2 shows that (A1) is a condition sufficient to ensure that Problem (P2) has a solution, but it is not necessary. The determination of conditions necessary and sufficient to ensure that Problems (P1) and (P2) have a solution remains an open problem.

2. In retrospect, we can expect that if Problems (P1) and (P2) have a solution, then the same function $\delta$ from $\Omega \times U$ to partial orders on the set $\mathscr{Z}$ of agents will solve both Problems (P1) and (P2). This happens because the sets $\Psi^{\prime}\left(\Psi^{\prime} \subset \Psi\right)$ and $M^{\prime}\left(M^{\prime} \subset M\right)$ are obtained by the same restriction on $\Psi$ and $M$, respectively. Specifically, $\Psi^{\prime}\left(M^{\prime}\right)$ consists of all the elements of $\Psi(M)$ that are $(\mathscr{B} \otimes \mathscr{U})$-measurable (see Section 3 and the definition of $M$ and $M^{\prime}$ ).

\section{Discussion}

It is possible to obtain results similar to those of Section 5 for particular designs $\gamma \in \Gamma$ that possess Property $\mathrm{CI}^{*}$ or Property $\mathrm{C}^{*}$. Consider the same information structure as in Section 4, a design $\gamma \in \Gamma$, and assume that $\gamma$ possesses Property CI* (respectively, Property $\left.C^{*}\right)$. Let $\Psi_{\gamma}^{*}$ (respectively, $\bar{\Psi}_{\gamma}^{*}$ ) be the set of maps $\psi^{*}: G^{\gamma} \rightarrow S_{N}$ such that $\gamma$ possesses Property CI* (respectively, Property C*). We wish to find a simple characterization of $\Psi_{\gamma}^{*}\left(\right.$ respectively, $\left.\bar{\Psi}_{\gamma}^{*}\right)$ in terms of a function $\delta^{*}$ (respectively, $\bar{\delta}^{*}$ ) from $G^{\gamma}$ to partial orders on the set $\mathscr{Z}$ of agents. More specifically, we are concerned with the following problems:

$(\mathrm{P} 1)^{*}$ Determine the conditions under which there is a function $\delta^{*}$ from $G^{\gamma}$ to partial orders on $\mathscr{Z}$ such that $\Psi_{\gamma}^{*}=M_{\gamma}^{*}$, where $M_{\gamma}^{*}$ is the set of all total order functions $\mu^{*}: G^{\gamma} \rightarrow S_{N}$ that are compatible with $\delta^{*}$.

$(\mathrm{P} 2)^{*}$ Determine the conditions under which there is a function $\bar{\delta}^{*}$ from $G^{\gamma}$ to partial orders on $\mathscr{Z}$ such that $\bar{\Psi}_{\gamma}^{*}=\bar{M}_{\gamma}^{*}$, where $\bar{M}_{\gamma}^{*}$ is the set of total or- 
der functions $\bar{\mu}^{*}: G^{\gamma} \rightarrow S_{N}$ that are compatible with $\bar{\delta}^{*}$ and have the following property: for all $s \in S_{k}$ and all $k=1,2, \ldots, N$,

$$
\begin{aligned}
{\left[P_{T_{k-1}^{k}(s)}\right]^{-1} } & \left(P_{T_{k-1}^{k}(s)}\left(T_{k}^{N} \cdot \bar{\mu}\right)^{-1}(s)\right) \\
& \in \mathscr{F}\left(T_{k-1}^{k}(s)\right) \cap\left[P_{T_{k-1}^{k}(s)}\right]^{-1}\left(P_{T_{k-1}^{k}(s)}\left(G^{\gamma}\right)\right) .
\end{aligned}
$$

Let $\mathscr{J}^{\gamma^{k}}$ be the information partition of agent $k$, induced by the control law $\gamma \in \Gamma, \gamma:=\left(\gamma^{1}, \gamma^{2}, \ldots, \gamma^{N}\right)$ (see (3.5)). We make the following assumption:

(A2) For each intrinsic event $(\omega, u) \in G^{\gamma}$ and for each agent $s_{m} \in \mathscr{Z}$ there is a unique smallest cardinality set $\Lambda_{*}^{s_{m}}(\omega, u) \subset \mathscr{Z}$ with the following properties:

$$
\begin{aligned}
& \mathscr{J}^{\gamma^{s_{m}}} \cap\left[\mathscr{P}_{\Lambda_{*}^{s m}(\omega, u)}\right]^{-1}\left(\mathscr{P}_{\Lambda_{*}^{s m}(\omega, u)}(\omega, u)\right) \\
& \subset\left\{\varnothing,\left[\mathscr{P}_{\Lambda_{*}^{s m}(\omega, u)}\right]^{-1}\left(\mathscr{P}_{\Lambda_{*}^{s_{m}}(\omega, u)}(\omega, u)\right)\right\} ; \\
& \mathscr{J}^{\gamma^{s m}} \cap\left[\mathscr{P}_{\mathscr{L}^{*}}\right]^{-1}\left(\mathscr{P}_{\mathscr{L}^{*}}(\omega, u)\right) \not \subset\left\{\varnothing,\left[\mathscr{P}_{\mathscr{L}^{*}}\right]^{-1}\left(\mathscr{P}_{\mathscr{L}^{*}}(\omega, u)\right)\right\}
\end{aligned}
$$

for any set $\mathscr{L}^{*} \subset \mathscr{Z}$ such that $\Lambda_{*}^{s_{m}}(\omega, u) \not \subset \mathscr{L}^{*}$.

Assumption (A2) is the design-dependent analogue of Assumption (A1) and has an interpretation similar to that of (A1).

Under Assumption (A2) we can show that there exists a function $\delta^{*}$ (respectively, $\left.\bar{\delta}^{*}\right)$ from $G^{\gamma}$ to partial orders on the set $\mathscr{Z}$ of agents that solves Problem $(\mathrm{P} 1)^{*}$ (respectively, Problem $\left.(\mathrm{P} 2)^{*}\right)$ using arguments similar to those of Section 5.

\section{Conclusions-Reflections}

Investigation of the interaction between the information structure and concurrency reveals, for each intrinsic event $(\omega, u)$, the information flow that results in a deadlock-free operation of the corresponding informationally decentralized nonsequential stochastic controlled system. Specifically, the sets $\Lambda^{s_{m}}(\omega, u)$, defined in Section 5, determine "who must communicate with whom along each intrinsic event" to avoid deadlock. The sets $\Lambda^{s_{m}}(\omega, u)$ are the nonsequential analogue of the field basis that is defined on p. 1559 of [11] and plays a fundamental role in the analysis of sequential stochastic controlled systems.

The design implications of Assumption (A1) (respectively, Assumption (A2)) are important and intuitive. Specifically, these assumptions make clear the intuition that if in any informationally decentralized nonsequential system it is desirable that a particular decision be immune to information losses, it is necessary that multiple nonintersecting sets $\Lambda^{s_{m}}(\omega, u)$ (respectively, $\Lambda_{*}^{s_{m}}(\omega, u)$ ) that possess Property (5.1) (respectively, Property (6.2)) and do not satisfy Assumption (A1) (respectively, Assumption (A2)) exist for each agent and each intrinsic event that includes the decision. Consider, for example, routing in a mobile network. If the objective is to transmit a message from Node A to Node B through the network, one would like to have more than one alternative route available, so that in the 
event anode along one of the routes fails the message can still reach its destination. This requirement implies that multiple nonintersecting sets $\Lambda^{s_{m}}(\omega, u)$ of mobiles, that possess Property (5.1) (respectively, Property (6.2)) but do not satisfy Assumption (A1) (respectively, Assumption (A2)), must exist for each mobile that is included in one or more of the routes. Thus, the results of this paper point out potential advantages of decentralized concurrent systems that have "informational redundancy," that is, they have, along each intrinsic event $(\omega, u)$ and for each agent $s_{m}$, multiple nonintersecting sets $\Lambda^{s_{m}}(\omega, u)$ with the properties stated above. The results of this paper also make clear that partial-order-based concurrency models are valid when every action can be traced to sets of predecessors actions that satisfy Assumption (A1) (respectively, Assumption (A2)). This last observation may be surprising to some. Computer scientists are accustomed to using partial orders to describe concurrency (see [7]). The results of this paper highlight the fact that such descriptions are not always correct.

\section{References}

[1] M. S. Andersland, Decoupling nonsequential stochastic control problems, Systems Control Lett., 16 (1991), 65-69.

[2] M. S. Andersland and D. Teneketzis, Information structures, causality, and non-sequential stochastic control, I: design-independent properties, SIAM J. Control Optim., 30(6) (1992), 14471475.

[3] M. S. Andersland and D. Teneketzis, Information structures, causality, and non-sequential stochastic control, II: design-dependent properties, SIAM J. Control Optim., 32(6) (1994), 17261751.

[4] P. Billingsley, Probability and Measure, 2nd edn., New York: Wiley (1986).

[5] C. Dellacherie and P. A. Meyer, Probabilities and Potential, North-Holland Mathematical Studies 29, Amsterdam: North-Holland (1978).

[6] W. S. Lai, Protocol traps in computer networks - a catalog, IEEE Trans. Comm. 30 (1982), 1434 1449.

[7] R. Langerak, E. Brinksma, and J.-P. Katoen, Causal Ambiguity and Partial Orders in Event Structures, Lecture Notes in Computer Science, 1243, Berlin: Springer-Verlag (1997), pp. 317 331.

[8] D. Teneketzis, On information structures and nonsequential stochastic control, CWI Quart. 9(3) (1996), 241-260, special issue on Systems and Control.

[9] J. von Neumann and O. Morgenstern, The Theory of Games and Economic Behavior, Princeton, NJ: Princeton University Press (1944), Chapter 2.

[10] H. S. Witsenhausen, On information structures, feedback and causality, SIAM J. Control, 9 (1971), 149-160.

[11] H. S. Witsenhausen, Separation of estimation and control for discrete time systems, Proc. IEEE, 59(11) (1971), 1557-1566.

[12] H. S. Witsenhausen, The Intrinsic Model for Discrete Stochastic Control: Some Open Problems, Lecture Notes in Economics and Mathematical Systems, 107, Berlin: Springer-Verlag (1975), pp. 322-335.

[13] H. S. Witsenhausen, Equivalent stochastic control problems, Math. Control Signals Systems, 1(1) (1988), 1-13. 